\title{
Honeybee Activity Monitoring in a Biohybrid System for Explosives Detection
}

\author{
Mitar Simić ${ }^{1}$, Ross Gillanders ${ }^{2}$, Aleksej Avramović ${ }^{\text {, Slavica Gajić }}{ }^{1}$, Vedran Jovanov- \\ ić$^{1}$, Vladan Stojnić ${ }^{1}$, Vladimir Risojević ${ }^{1}$, James Glackin ${ }^{2}$, Graham Turnbull ${ }^{2}$, Janja \\ Filipi $^{3}$, Nikola Kezić ${ }^{4}$, and Zdenka Babić ${ }^{1}$ \\ ${ }^{1}$ University of Banja Luka, Faculty of Electrical Engineering, Patre 5, 78000 Banja Luka, Bos- \\ nia and Herzegovina \\ ${ }^{2}$ School of Physics and Astronomy, University of St Andrews, North Haugh, KY16 9SS St. \\ Andrews, United Kingdom \\ ${ }^{3}$ University of Zadar, Department of Ecology, Agronomy and Aquaculture, Trg kneza Višesla- \\ va 9, 23000 Zadar, Croatia \\ ${ }^{4}$ University of Zagreb, 10000 Zagreb, Croatia
}

\begin{abstract}
Free-flying honeybees can electrostatically collect particles from air in the flying and foraging areas, which in conjunction with organic-based explosive vapor sensing films, placed at the entrance to the beehive, can be used as a passive explosive sensing mechanism. Moreover, bees can be trained to actively search for a smell of explosive. Using trained honeybees in conjunction with a system for honeybee localization enables generation of a spatial-time honeybee density map, where the most visited places point to suspicious areas. In both methods (passive and active), bees' activity monitoring plays a significant role, providing information about environmental parameters and activities of bees at the entrance and exit of a beehive. In this paper we present the design and realization of an electronic system for bee activity monitoring at the front of a hive while using bees for explosive detection. The system also monitors air temperature and relative humidity. Results obtained to date from activity monitoring are useful in planning testing activities within our active and passive method, as it can determine the optimal period of the day and environmental parameters in which bees are most active.
\end{abstract}

Keywords: Biosensors, explosive detection, honeybees, organic-based explosive vapor sensing films, UAV.

\section{Introduction}

Detection of legacy landmines is a very important step in humanitarian mine clearance (demining) which is a pressing concern in many countries $[1,2]$. It is estimated that today there are still tens of millions of anti-personnel mines in the ground in over 60 countries [3]. According to the United Nations, approximately 2,000 people are

adfa, p. 1, 2011.

(C) Springer-Verlag Berlin Heidelberg 2011 
killed or injured by landmines every month. Over half the landmine casualties are civilians [4].

Current humanitarian mine detection and demining methods mainly include: (1) manual detection with a metal detector, (2) detection by specially trained dogs or rats, and (3) mechanical clearance. Each of these methods has some advantages over others, but in general the main disadvantages of all three are high cost, time-consuming training procedures, and the risks and dangers for involved personnel. Thus, there are ongoing demands for new technologies which may provide effective demining additional method to conventional methods.

Our research within the project "Bees for explosive detection (Bee4Exp)" funded by NATO Science for Peace and Security Programme is directed towards the use of honeybees as biosensors for legacy landmine detection. Honeybees are known to have an excellent sense of smell, which they use to find the food. Training honeybees to smell explosives (e.g. TNT and DNT odors), enables us to actively use them to search for landmines. With a few days' conditionig in a mesh tent, bees associate the scent of TNT and DNT with food (sugar solution). The conditioned honeybee colony is then transferred to the mine field to search for TNT and DNT odors. So, bees can passively collect particles from the air while free-flying in foraging areas, and can be trained to actively search for smell of TNT and DNT -these two options are exploited for the development of methods for the use of bees in explosive detection. In the active method we monitor bees over a minefield and find the most frequently visited places, which then could be marked as suspected mine-contaminated areas. Our approach is based on an Unmanned Aerial Vehicle (UAV), high definition georeferenced video capturing images in both the visible and infrared spectrum, and sophisticated video analysis algorithms [5-6]. The passive method is based on the fact that during foraging honeybees are collecting particles and odors from surrounding. We are using bees applied to free fly on test mine fields in order to collect TNT and DNT particles which they are accumulating in the hive. The main advantages of using bees include the short period required for conditioning, the ability to cover a wide area in large numbers, and using honeybees for explosive detection is safer and cheaper additional method to the conventional methods [5].

However, well-known restriction in the use of bees is low temperature (below $\left.15^{\circ} \mathrm{C}\right)$ [2]. Wind and rain can also interfere with the activity of bees. Investigation of the influence of other environmental parameters is very important for understanding bee behavior in the field. In this paper we present an electronic system for bee activity monitoring which should provide information about environmental parameters with a time and date stamp, along with detected activity of bees at the entrance and at the exit of the beehive. Such information can be very useful in planning testing activities within our active and passive method, as it can determine the optimal period of the day and environmental parameters in which bees are most active.

This paper is organized as follows: in Section 2 the passive and active methods of using bees as biosensors for explosive detection are described. In Section 3 the electronic system for bee activity monitoring is presented. The main experimental results of testing the hardware-based prototype of the electronic system is presented in Sec- 
tion 4. Conclusion remarks and discussion related to our future work are given in Section 5 .

\section{Honeybees for Explosive Detection}

\subsection{Passive method}

For trace collection of polluting materials like explosives in the environment, a method known as "preconcentration" can be used [7-10]. Preconcentrator materials typically have a certain affinity to a specific, or class of, compounds, allowing them to be sorbed to the preconcentrator surface over time. The sorbed material can then be thermally desorbed to deliver a much higher concentration of analyte to the sensing probe than can normally be detected in real time.

Organic semiconductor films have been used as optical explosive sensors in recent years owing to their solution processing, reversibility, sensitivity and spectroscopic properties [11-14]. These films emit light in the yellow when photoexcited by blue light, and this strong emission can be quenched by trace amounts of explosives, leading to a characteristic decrease in the peak intensity.

As mentioned above, free-flying, foraging honeybees are known to electrostatically collect explosives and other materials on their body hair. By using preconcentrators to sorb the explosives from the returning bees, the presence of landmines in the area may be reliably detected, leading to faster and safer surveying for landmine-contaminated land.

A fluoropolymer, Aflas (AGC Chemicals), was dissolved in tetrahydrofuran and spot- or blade-coated onto a paper or canvas substrate, respectively, and allowed to dry. For collecting the explosives from the honeybee body hair, the canvas substrates were inserted into $1 \mathrm{~cm}^{2}$ tubes made of multiwall polycarbonate sheets, four for insertion in the entrance and four for insertion in the exit. This allowed the separation of returning bees and those leaving the colony. The paper-based substrates were also used to sample the colony air by placing the substrate in the nozzle of an air sampler, placing the nozzle into the hive via a specialized cupola, and sampling for 10 minutes at $60 \mathrm{Lmin}^{-1}$.

The passive method, in contrast to the tent-based training of the active method, allows the honeybees to free-fly during standard foraging activity in a given area and return to the hive. Typically control samples are taken on a known non-contaminated site over 24 hours before the colonies are moved to the test site. The cartridges containing the four preconcentrators are placed in the colony for 24 hours, prior to collection, sealing in an airtight bottle, and subsequent thermal desorption and optical detection.

To analyse the field samples, the commercial organic semiconductor Super Yellow (Merck) was dissolved in toluene and spin-coated onto clean glass slides. The sensor film was placed in a chamber containing the preconcentrator and a heating element to allow the preconcentrator to desorb the explosives. The heating element was ramped to $100^{\circ} \mathrm{C}$ over approximately 90 seconds, and the sensor film excited by a $405 \mathrm{~nm}$ 
laser from Photonic Solutions. The emission was monitored for 300 seconds with a fibre-couple spectrometer.

Our test site was at Benkovac, Croatia during September 2018. Benkovac test site, inaugurated in 2000, has 1000 buried mine targets in a Mediterranean climate over $10,000 \mathrm{~m}^{2}$. There are 39 blind test lanes each $47 \mathrm{~m}$ long, $1 \mathrm{~m}$ wide, and various mines are buried at 5-27 cm depth. Distance between the lanes is $3 \mathrm{~m}$. The lanes are divided into $1 \mathrm{x} 1 \mathrm{~m}$ squares.

Initial results from the passive method indicate Aflas is a good preconcentrator material for explosives. The emission from the Super Yellow film shows a decrease of around $15 \%$ when $50 \mathrm{ng}$ of DNT is desorbed from a preconcentrator substrate under laboratory conditions compared to the control experiment where around $1 \%$ of emission is lost over the same time period due to photo-oxidation of the sensor film, as shown in Fig. 1.

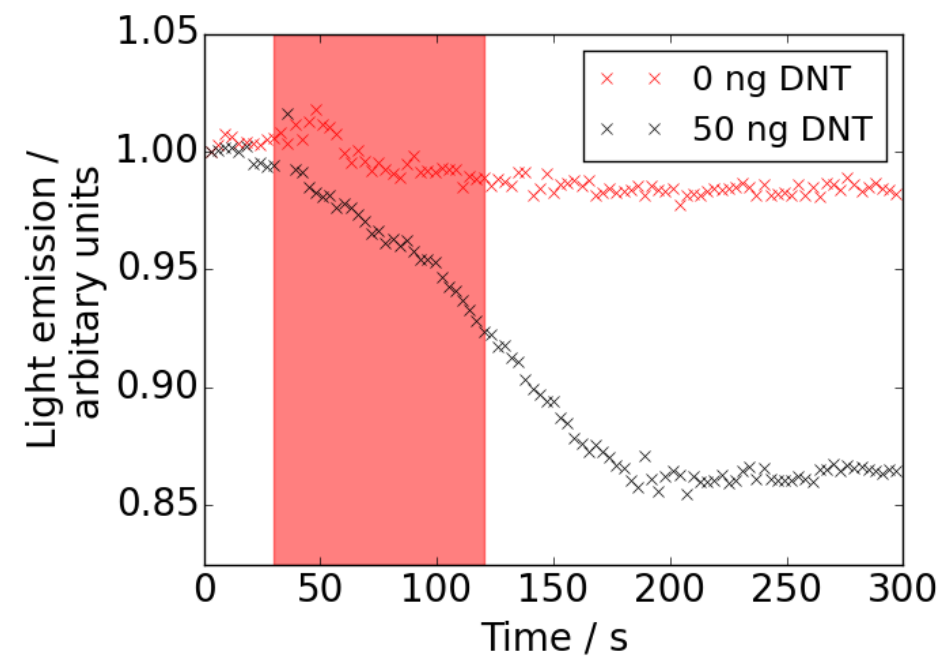

Fig. 1 Decrease in Super Yellow emission from a DNT-exposed Aflas preconcentrator

\subsection{Active method}

The active method for using honeybees as bio sensors implemented in this project refers to monitoring the activity of honeybees, over a field which is suspected to be contaminated with mines. Trained honeybees are expected to sense the smell of explosives as a food, and the active method has a goal to tracked honeybees on a field and to determine exact spots where are they gathered. It is important to emphasize that this method is primarily intended for safety and quality control.

The proposed method is carried out in two phases. The first one includes the specially designed preparation of honeybees so they would react on explosive vapors of explosive substances contained in landmines. The goal is to train honeybees to recognize explosives as food. It training is successful; honeybees will show behavior around the landmines similar as around the food, since they will associate scent of 
explosive with the food belonging to a certain area where the desired minefield is located. The second phase includes honeybee's tracking and tracing over the inspected area, in order to pinpoint exact locations where are they gathered. Detected locations with most frequent honeybee's occurrences are most suspicious to contain unexploded ordnances. In order to detect honeybees during their flight over an area, features which provide good detection possibility of honeybees must be extracted, either from visual spectrum or infrared spectrum.

The important preparation step includes setting up the data acquisition parameters correctly. Having in mind that honeybees can cover a large area during the flight outside a hive, it is impossible to track and trace each individual honeybee. An approach used in our experiments relied on high-resolution video capturing of part of the field where we can expect their major presence (near a hive, or around a feeder). Captured data should provide enough information for automatic motion detection of small objects and estimation of the most frequent places of occurrences. We used UAV equipped with an ultra-high definition video (UHD) and a thermal camera (TC). To avoid strong winds coming from the UAV's propellers, an UAV should hover at least 8 to 10 meters above the ground. It that case, winds won't distract honeybees, although they can cause additional movement of small objects on the ground (leaves, branches, etc.). For example, UHD camera equipped with $50 \mathrm{~mm}$ lens, will cover an area around 7 meters in width and 4 meters in height and will use more than 8 million pixels to store the data for one frame only. Considering the size of an individual honeybee (about $1 \mathrm{~cm}$ ) each honeybee covers the part of the frame of size around 20 pixels. Beside usual RG cameras, a good choice of a sensor is thermal camera, since the temperature of a flying honeybee is usually $35^{\circ} \mathrm{C}$. Currently available TCs are rather low-resolution and expensive but offer a good (thermal) information, which can be used to enhance UHD video. Nevertheless, a combination of visual and thermal features should provide more useful data for automatic detection of honeybees and easier detection of other moving objects of no interest (such as grass, plants, sticks, branches, etc.).

After the video is captured, a large amount of data is collected and must be processed to come up with the final results. Since video must be captured from multiple locations and/or from multiple sensors (for example three UAV with cameras), the exact determining of actual coordinate is very important. So, system navigation is another crucial aspect of the active method. Standard satellite navigation can provide the positioning accuracy of couple of meters, which can be reduced with further postprocessing. If possible, it is recommended to use Real-Time Kinematic GPS (RTK), which provides much greater accuracy than standard GPS and overall accuracy under $2 \mathrm{~cm}$. Basically, the proposed capturing system must contain UAV with accurate GPS, and UHD and TC sensors, but additional sensors can be considered as well.

Considering that honeybees are very small insects (in average $1 \mathrm{~cm}$ long) and that their natural color makes them hard to detect when flying above grass- or dirt- terrain, the complete and final solution for their tracking and tracing is still missing. By analysis of a huge amount of captured video, we concluded that most descriptive feature that can separate honeybees from the background is their style of movements. Therefore, our research methodology is mostly based on state-off-the-art algorithms like 
Gaussian mixture model and Convolutional Neural Networks. Next, we implemented several case-specific methods for preprocessing (image filtering, video stabilization, etc.) and postprocessing (morphological operations, noise removal, etc.). The overall result of several overflights is a spatio-temporal occurrence density map (Fig. 2). This maps shows the most frequently visited places, which can indicate the possible locations of legacy landmines and other unexploded ordnances.

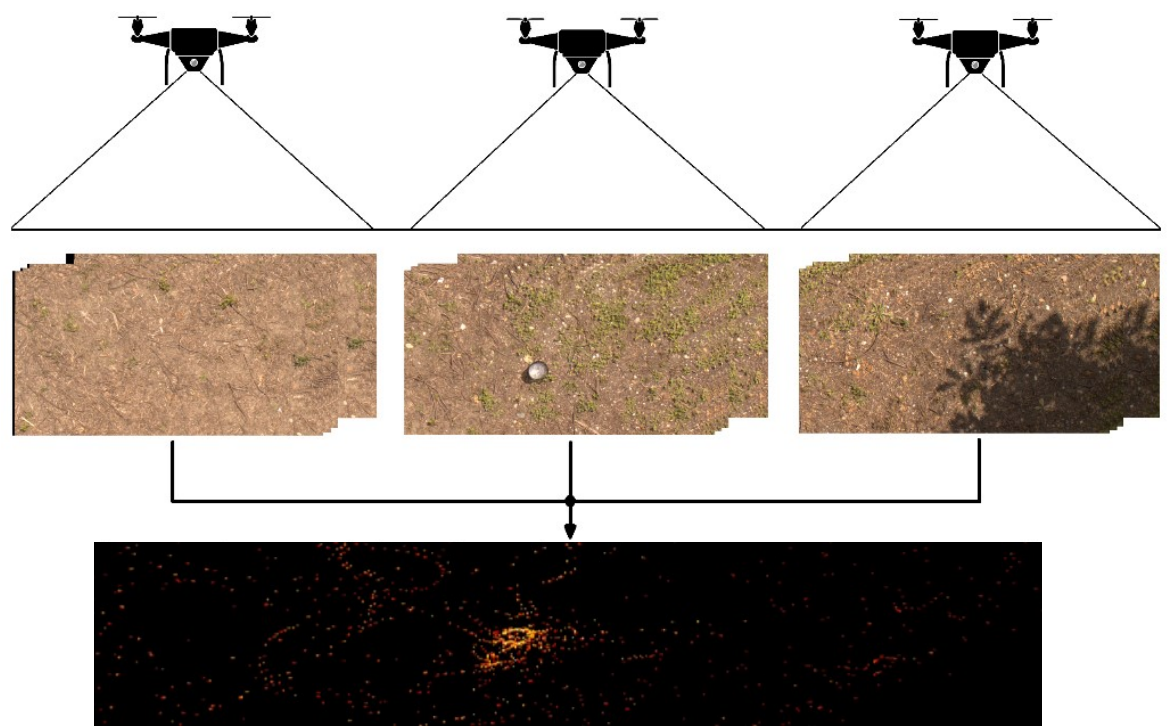

Fig. 2 Schematic representation of the active method.

\section{$3 \quad$ Electronic system for bees' activity monitoring}

The schematic overview of our electronic system for bee activity monitoring is shown in Fig. 3. We use an infrared (IR) LED as a source and a phototransistor in switching mode as a detector. The IR LED emits a light beam and if there is no bee in the input channel the phototransistor will be off, which can be detected by a voltage measurement between the OUT pin and the ground (GND). If the phototransistor is off, the expected value of voltage at the OUT pin is equal to the Vdd. However, if a bee is present in the input channel she will reflect light towards the phototransistor which will go to the on state, and voltage at the OUT pin will drop to the collector-emitter saturation voltage. A Microcontroller ( $\mathrm{uC}$ ) is used for voltage measurement with an embedded analog-to-digital converter (ADC). Monitoring the daily activities of bees is registered with information regarding time and date collected with a real time clock (RTC), as well as ambient temperature and relative humidity (RHT sensor). 


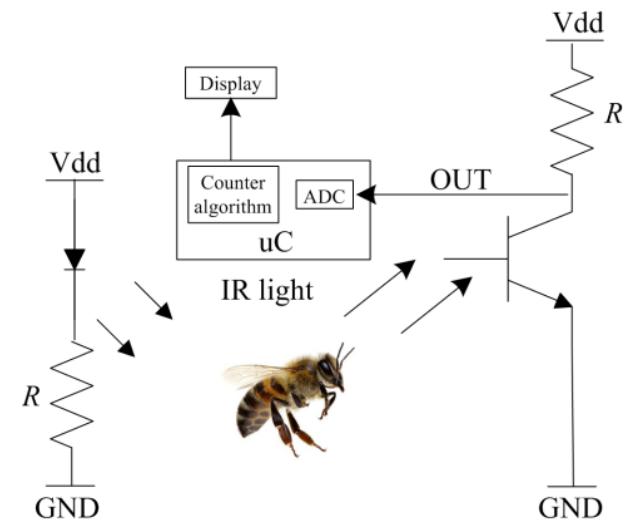

Fig. 3 Schematic overview of proposed system for monitoring of bees' activities

\section{Experimental results}

Hardware realization of the electronic system for bee activity monitoring (Fig. 4) is based on an ATmega2560 microcontroller, 6 QTR Pololu pairs of IR LED and phototransistor, DS3231 RTC and a Sensirion SHT11 RHT sensor. Tubes used for placement of the canvas substrates were used for Pololu sensors as well. Additionally, black tape was used to prevent penetration of daylight (includes also infrared wavelengths) which can cause faulty turning on of phototransistors.

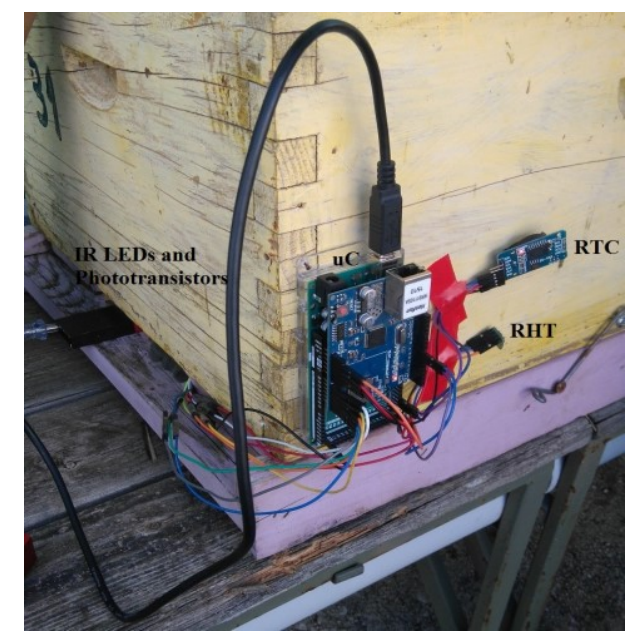

Fig. 4 Hardware realization of the electronic system for bee activity monitoring

The electronic system for bee activity monitoring was tested at the same time as the passive method at the Benkovac test site during September 2018. One small part of obtained results in monitoring of ambient air temperature $\left({ }^{\circ} \mathrm{C}\right)$, relative humidity 
$(\% \mathrm{RH})$ and percentage of input voltage compared to the Vdd are shown in Fig. 5 and Fig. 6. Results are presented starting from $5 \mathrm{am}$, with samples obtained everysecond. As shown in Fig. 5, it can be noticed that around 6:30 am bees started leaving the beehive along with the increase of temperature and decrease in relative humidity.
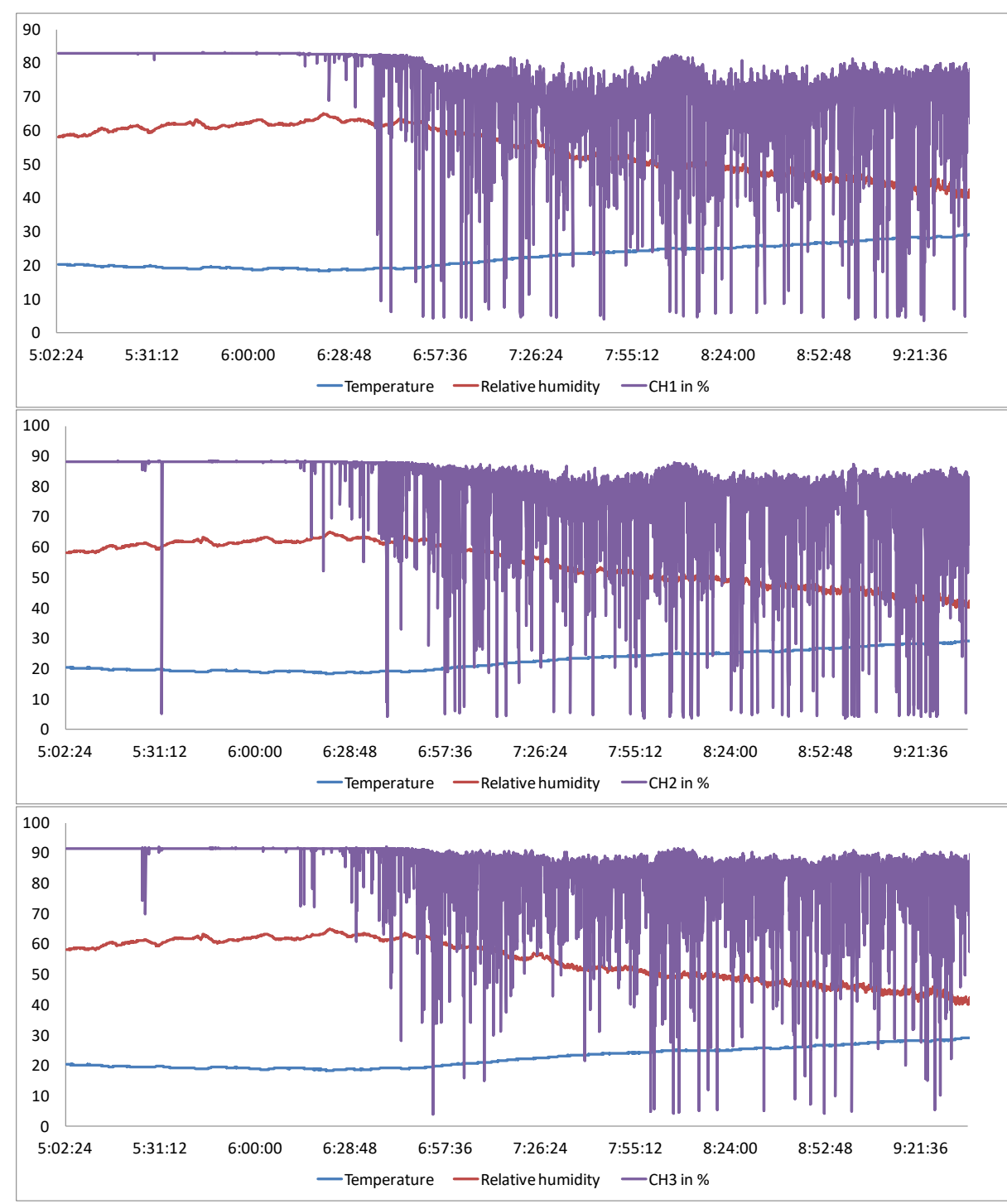

Fig. 5 Obtained results for channels at the exit of the hive

However, channels at the entrance of the beehive (Fig. 6) registered lower level of activities, which was expected as bees early in the morning start going out to look for food. 

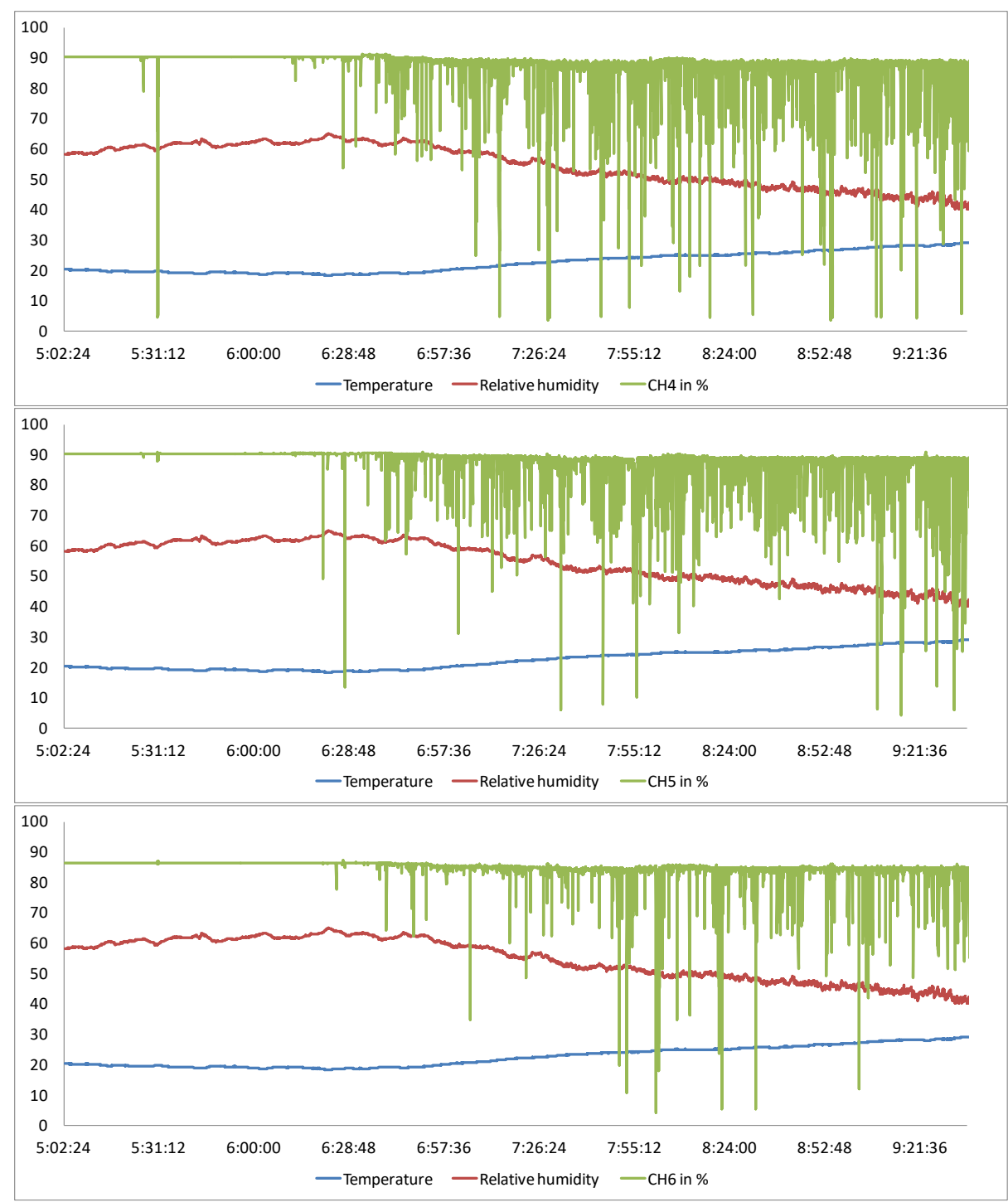

Fig. 6 Obtained results for channels at the entrance to the hive

However, the inverse behavior was registered at the end of the day- relative humidity was increasing while temperature and bee activities were decreasing.

Monitoring over longer periods of time during changes of environmental parameters can be very useful in planning testing activities and interpreting data within our active and passive methods, as it can determine optimal period of the day or ambient parameters in which bees are most active. 


\section{Conclusion}

In this paper we presented an electronic system for monitoring bees' activity. Our approach is to monitor environmental parameters as well as the detection of bees which are going out from the beehive to look for food. Such an electronic system is very important for our research for explosive detection with bees. Experimental results showed that both our approaches are promising with great potential for highimpact delivery of this research.

Obtained results showed that our active approach can be used for detection, tracking and tracing of honeybees, therefore for monitoring of their activity. Further work will include mathematical modeling of honeybee's occurrence and validation based on expert's opinion.

The promising early results from the passive method of collecting and subsequently detecting explosives in the environment indicate that free-flying honeybees can electrostatically collect enough explosive materials from the environment to be concentrated onto a fluoropolymer substrate and be detected by optical sensors. Efforts are ongoing to increase the sorbing affinity and detection sensitivity of the system.

As the future work, we are directed towards further research regarding electronic systems for bee activity monitoring. We have recognized the need for development of an algorithm for bee counting based on the measured voltage, as well as expansion of environmental parameters which can be monitored, such as direction and wind speed, UV level, or presence of volatile organic compounds.

\section{Acknowledgment}

This research is partially supported by the "Biological Methods (Bees) for Explosive Detection" international project, supported by NATO Science for Peace and Security (SPS) Programme, project number SPS 985355, and Ministry of Science and Technology Republic of Srpska, Bosnia and Herzegovina, "UAV Video Analysis in Biological Methods for Explosive Detection", project number 19/6-030/3-2-21-1/17.

\section{$7 \quad$ Conflicts of Interest}

The authors declare no conflict of interest.

\section{References}

1. Zdenka Babic, "Signal Processing Challenges in Biohybrid System Design," In Proceedings of the IEEE $7^{\text {th }}$ International Conference on Reliability, Infocom Technologies and Optimization (ICRITO'2018) (in press), invited paper, Noida, India, August 2018 
2. N. Kezić, Z. Babić, J. Filipi, G. Turnbull, R. Gillanders, J. Glackin, G. Mirjanić, M. Muštra and N. Pavković, "Bees for Explosive Detection", In Proceedings of 15th International Symposium Mine Action 2018, Slano, Croatia, April 9-12, 2018, pp. 42-44.

3. http://marshall-legacy.org/about-2/landmine_facts/ (accessed on 10.12.2018.)

4. https://research.un.org/en/mines/un-documents (accessed on 10.12.2018.)

5. A. Avramović, V. Jovanović, R. Pilipović, V. Stojnić, V. Risojević, S. Gajić, M. Simić, I. Ševo, M. Muštra, Z. Babić and J. Filipi, "Automatic monitoring of honeybees' activity outside of the hive from UHD video", In Proceedings of 14th Symposium on Neural Networks and Applications (NEUREL), Belgrade, Serbia, November 20-21, 2018.

6. A. Avramović, R. Pilipović, V. Stojnić, V. Jovanović, I. Ševo, M. Simić, V. Risojević and Z. Babić, "Honeybees video-tracking for explosive detection", In Proceedings of 15 th International Symposium Mine Action 2018, Slano, Croatia, April 9-12, 2018, pp. 45-48.

7. O. B. Egorov, M. J. O'Hara, J. W. Grate, Equilibration-based preconcentrating minicolumn sensors for trace level monitoring of radionuclides and metal ions in water without consumable reagents, Analytical Chemistry, 2006, 78 (15), 5480-5490.

8. K. Cizek, C. Prior, C. Thammakhet, M. Galik, K. Linker, R. Tsui, A. Cagan, J. Wake, J. La Belle, J. Wang, Integrated explosive preconcentrator and electrochemical detection system for 2,4,6-trinitrotoluene (TNT) vapor, Analytica Chimica Acta, 2010, 661 (1), 117 121.

9. M. Camara, F. James, P. Breuil, C. Pijolat, D. Briand, N. F. de Rooij, MEMS-based porous silicon preconcentrators filled with Carbopack-B for explosives detection, In 28th European Conference on Solid-State Transducers, Sberveglieri, G.; Ferrari, V., Eds. 2014; Vol. 87, pp 84-87.

10. N. Tiwary, M. Vinchurkar, M. Patel, R. Nathawat, S. Pandey, V. R. Rao, Fabrication, Characterization and Application of $\mathrm{ZnO}$ Nanostructure-Based Micro-Preconcentrator for TNT Sensing, Journal of Microelectromechanical Systems, 2016, 25 (5), 968-975.

11. S. J. Toal, W. C. Trogler, Polymer sensors for nitroaromatic explosives detection, Journal of Materials Chemistry, 2006, 16 (28), 2871-2883.

12. S. W. Thomas, G. D. Joly, T. M. Swager, Chemical sensors based on amplifying fluorescent conjugated polymers, Chemical Reviews, 2007, 107 (4), 1339-1386

13. R. N. Gillanders, I.D.W. Samuel, G. A. Turnbull, A Low-Cost, Portable Optical Explosive-vapour Sensor, Sensors and Actuators B-Chemical, 2017, 245, 334-340.

14. N. Bolse, R. Eckstein, M. Schend, A. Habermehl, C. Eschenbaum, G. Hernandez-Sosa, U. Lemmer, A digitally printed optoelectronic nose for the selective trace detection of nitroaromatic explosive vapours using fluorescence quenching, Flexible and Printed Electronics, 2017, 2 (2), 024001. 